\title{
Understanding graphene's role as a protective substrate for atomic-resolution electron microscopy of small organic molecules
}

Blanka Janicek $^{1}$, Priti Kharel $^{2}$, Sang hyun Bae $^{2}$ and Pinshane Huang ${ }^{2}$

${ }^{1}$ University of Illinois at Urbana-Champaign, Urbana, Illinois, United States, ${ }^{2}$ University of Illinois at Urbana-Champaign, United States

At one atom thick, graphene substrates are the ultimate low-background support membrane for (scanning) transmission electron microscopy. Graphene is also impermeable, electrically and thermally conductive; as a result, it increases the dose resistance of the materials it supports while minimizing background contrast [1], making it an ideal substrate for imaging beam-sensitive materials [2-4]. In particular, graphene can play a critical role in enabling high-resolution studies of soft materials and molecules, which are exceptionally beam sensitive with typical critical doses between $0.5-1000 \mathrm{e}^{-} / \AA^{2}[5]$. In these systems, graphene substrates have been shown to enable atomic-resolution imaging of monolayers of small organic molecules [6-7]. However, the mechanisms through which graphene protects organic molecules, as well as the magnitude of benefit provided by graphene substrates, have not been studied in detail. Here, we systematically investigate the protection factor that graphene substrates provide for small organic molecules and how it varies with experimental factors such as the accelerating voltage, dose rate, temperature, sample geometry, and molecular species. We use these data to interrogate the interactions between the electron beam, organic molecules, and graphene, as well as to determine optimal experimental conditions for high-resolution imaging of organic molecules.

We use selected area electron diffraction (SAED) to measure the critical doses of various small molecules, such as porphyrins and phthalocyanines, on graphene and compare them to conventional amorphous carbon (a-C) substrates. For example, Figure 1 shows data taken on a metalated porphyrin,cobalt(II) meso-tetrakis(4-methoxyphenyl) porphyrin (CoTMPP). Figure 1b shows a typical SAED pattern of bilayer CoTMPP on graphene at 1 and $200 \mathrm{e}^{-} / \AA^{2}$, demonstrating the rapid decay of diffraction spot intensity. Overall, we find that graphene can provide significant protection, measuring up to $7 \mathrm{x}$ improvement in critical dose (Figure 1c). Notably, at $300 \mathrm{kV}$, graphene's protection factor is higher than that obtained by cryogenic cooling to $96 \mathrm{~K}$ (Figure 1d) and increases as a function of scattering vector $\mathrm{k}$, indicating that graphene substrates can be an effective means of preserving the high-frequency information needed for atomic-resolution imaging. However, we also find graphene's protection factors are strongly dependent on the experimental conditions, measuring only $2-3 \mathrm{x}$ at $80 \mathrm{kV}$ (Figure $1 \mathrm{~d}$ ). We attribute this effect to the increased role of radiolysis and secondary damage mechanisms at low accelerating voltages. Overall, our studies illustrate how to take advantage of the complex electron-sample interactions of molecules on graphene substrates for atomic-resolution imaging of extremely beamsensitive samples [8]. 
a
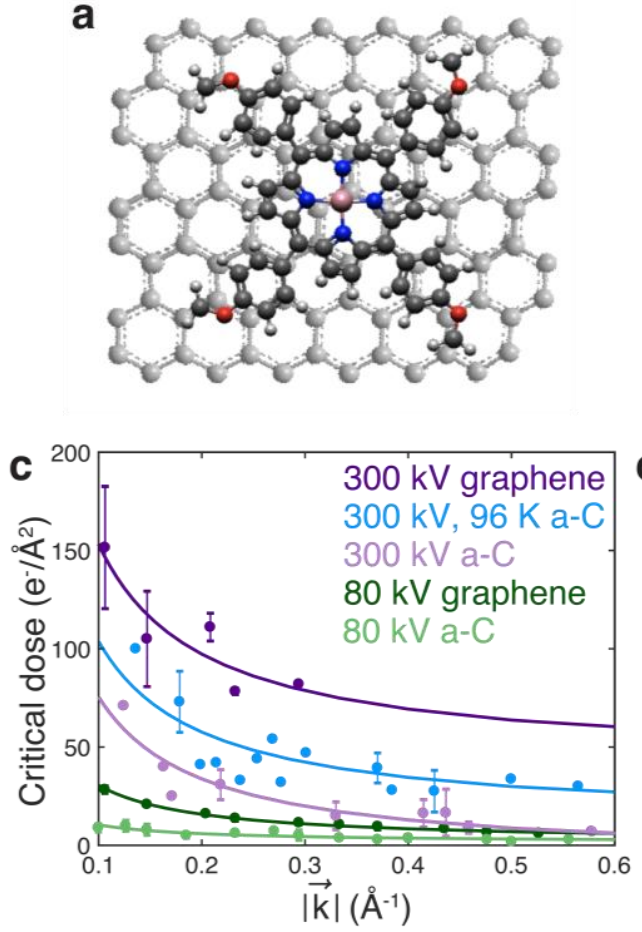
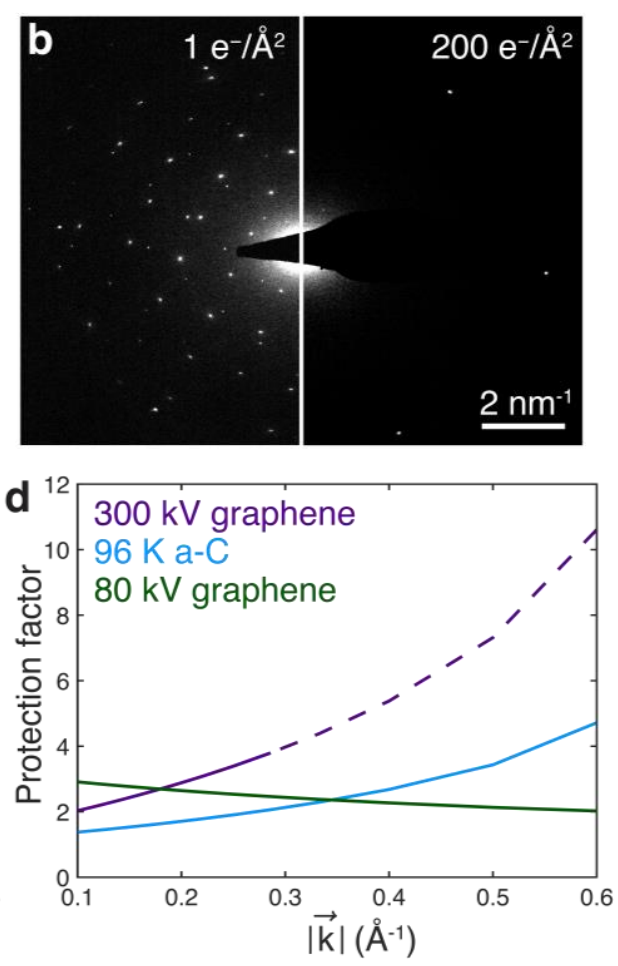

Figure 1. Trends in critical dose and protection factor with scattering vector $\mathrm{k}$ for CoTMPP under a variety of beam and sample conditions, including temperature and accelerating voltage. (a) Cartoon structure of CoTMPP on graphene. (b) Representative $80 \quad \mathrm{kV}$ SAED pattern of bilayer CoTMPP on graphene at 1 (left) and 200 (right) e-/Å2. After $200 \quad \mathrm{e}-/ \AA 2$, the remaining visible diffraction spots are from the graphene substrate. (c) Plot comparing critical dose vs scattering vector $\mathrm{k}$ measured for a variety of beam and sample conditions. At $300 \mathrm{kV}$ we compare $2 \mathrm{D}$ crystals on graphene substrates at room temperature (dark purple), bulk crystals on a-C at room temperature (light purple), and bulk crystals on a-C at $96 \mathrm{~K}$ (blue). At $80 \mathrm{kV}$ we compare $2 \mathrm{D}$ crystals on graphene substrates at room temperature (dark green) and bulk crystals on a-C at room temperature (light green). All the data (points) are shown with a linear fit to the mean. (d) The protection factor of graphene at 80 (green) and 300 (purple) $\mathrm{kV}$ versus scattering vector $\mathrm{k}$, obtained by taking a ratio of the graphene to a-C best fit curves from the data in (d). We also plot the cryogenic cooling protection factor (blue), obtained by taking a ratio of the a-C $96 \mathrm{~K}$ and room temperature best fit curves from the data in (d). Solid lines indicate data obtained from the measured range of k-vectors, while dashed lines are an extrapolation.

\section{References}

[1] R Pantelic et al., Solid State Commun. 152 (2012), p. 1375.

[2] G Algara-Siller et al., Appl. Phys. Lett. 103 (2013), p. 203107.

[3] B Janicek et al., Nano Letters 19 (2019), p. 6308.

[4] Y Han et al., PNAS 117 (2020), p.1009.

[5] R Egerton, et al., Micron 119 (2019), p. 72.

[6] P Kharel et al., submitted Jan 2021.

[7] A Mittleberger et al., Sci. Rep. 8 (2018), p. 6.

[8] This work was supported by the Packard Foundation and an NSF CAREER award under DMR1846206. The experimental work was carried out in the Materials Research Laboratory. 